\title{
Assessment of pain
}

\section{Knowledge, attitudes, and practices of health care providers in Almadinab Almunawwarah, Saudi Arabia}

Khalid W. Al-Quliti, MBBS, MD, Majed S. Alamri, $P h D, R N$.

\section{ABSTRACT}

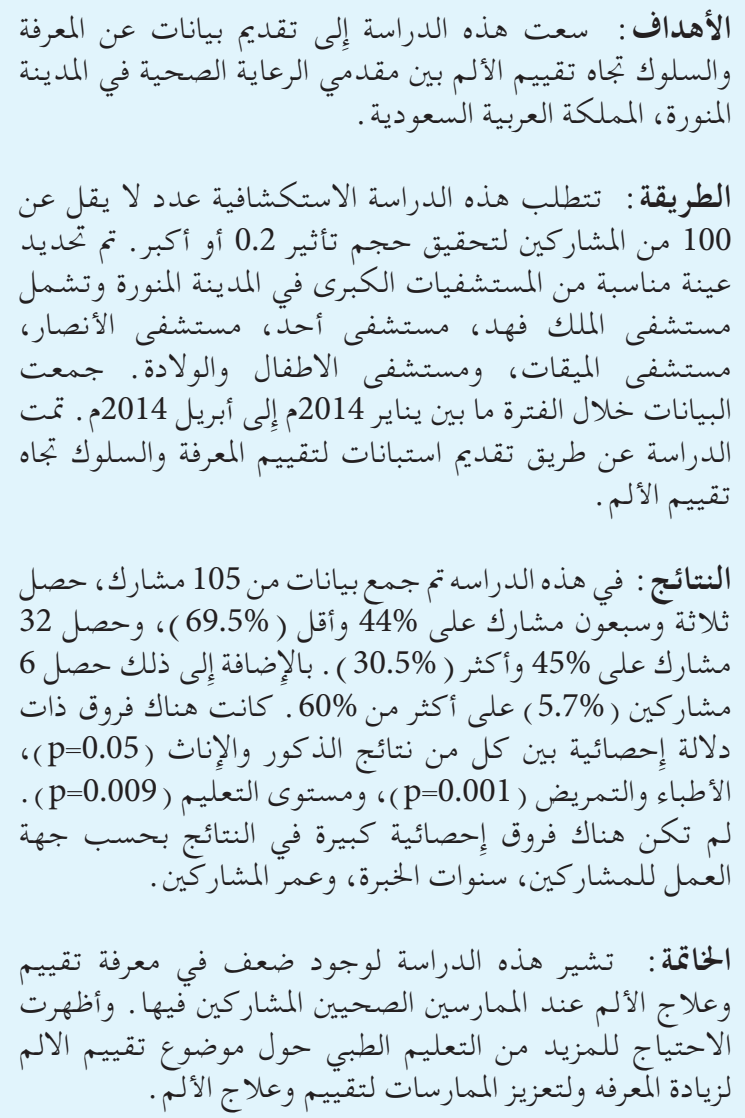

Objective: To present data on knowledge and attitudes toward pain assessment among health care providers in Almadinah Almunawwarah, Saudi Arabia.

Methods: This exploratory study required at least 100 participants to attain an effect size of 0.2 or larger. A conveniencesamplewasrecruitedfrom themajorhospitals in Almadinah Almunawwarah, Saudi Arabia; King Fahad
Hospital, Ohud Hospital, Alansar Hospital, Almeqat Hospital, and the Maternity Hospital. Data collection occurred between January and April 2014. Knowledge of pain assessment was examined by administering the Knowledge and Attitudes Survey Regarding Pain.

Results: Data from 105 participants was collected. Seventy-three participants scored $44 \%$ or below $(69.5 \%)$, and 32 participants scored $45 \%$ and above (30.5\%). Additionally, only 6 participants (5.7\%) scored above $60 \%$. There were significant differences between male and female scores $(p=0.05)$, physicians' and nurses' scores $(p=0.001)$, and level of education $(p=0.009)$. There were no significant differences in the passing scores across means of nationality, the department where participants worked, years of experience, and age of participants.

Conclusion: There is a deficit in pain assessment knowledge, and pain management in the study group. Continuous education on pain assessment is required to increase the health care providers' knowledge, and enhance their practices regarding pain assessment and treatment.

Neurosciences 2015; Vol. 20 (2): 131-136 doi: 10.17712/nsj.2015.2.20140546

From the Section of Neurology (Al-Quliti), Department of Medicine, College of Medicine, Taibah University, Almadinah Almunawwarah, and the Department of Nursing (Alamri), College of Applied Medical Sciences, Majmaah University, Majmaah, Kingdom of Saudi Arabia.

Received 27th August 2014. Accepted 9th March 2015.

Address correspondence and reprint request to: Dr. Khalid W. Al-Quliti, Chairman, Section of Neurology, Department of Medicine, College of Medicine, Taibah University, Almadinah Almunawwarah, Kingdom of Saudi_Arabia.E-mail:kh_alquliti@yahoo.com

Disclosure. The authors declare no conflicting interests, support or funding from any drug company. 
$\mathrm{P}$ ain is subjective and can differ from one person to another. Pain assessment can be a simple process by assessing location and intensity of pain. ${ }^{1}$ Failing to assess pain may affect quality of life, and increase the length of stay of hospitalized clients. ${ }^{2}$ More than 116 million Americans have pain that persists for weeks to years, and pain costs $\$ 560-635$ billion per year. ${ }^{3}$ The International Association for the Study of Pain ${ }^{4}$ defines pain as "an unpleasant sensory and emotional experience associated with actual or potential tissue damage, or described in terms of such damage." It is crucial that health care providers assess pain with accurate methods that reflect the intensity of the client's pain. Pain assessment is considered a fifth vital sign; many institutions nationally and internationally apply policies to include pain assessment in each patient's chart, along with vital signs. Pain has several classifications; common categories are: acute pain, chronic pain, and cancer pain. Pain is also classified by mode of origin and transmission to nociceptive pain, somatic and visceral pain, neuropathic pain, dysesthetic pain, and lancinating pain. These classifications help health care providers to choose the appropriate management plan for pain relief. 5 There are several validated assessment tools in the literature to assess the intensity of pain; for example, the Numeric Rating Scale (NRS), Visual Analog Scale, Verbal Descriptor Scale, and Wong-Baker Faces Scale. ${ }^{6,7}$ For critically ill adults who cannot communicate properly, there are several validated tools including the, Behavioral Pain Scale, Critical-Care Pain Observation Tool, and Face, Legs, Activity, Cry, and Consolability (FLACC) pain scale. ${ }^{6}$ The Association of Critical-Care Nurses ${ }^{6}$ issued a practice alert recently regarding pain assessment in critically ill adults. This report emphasizes: the need for patients to self-report pain using a validated pain assessment tool; the necessity of using a validated behavioral pain scale for critically ill adult clients who cannot communicate, avoiding referring primarily to vital signs for pain assessment of critically ill adult clients, and, for those unable to communicate, asking someone who knows the client well to identify any behavior that may indicate pain. Furthermore, the implementation of a nonverbal pain scale in critical care units improved clients' ratings of their pain experience, improved documentation by nurses, and increased nurses' confidence in assessing pain in nonverbal clients. ${ }^{8,9}$ From clinical practice in different Ministry of Health hospitals in Almadinah Almunawwarah, Saudi Arabia, we noticed that there are no specific protocols for pain assessment or pain management. Additionally, there is a scarcity of literature addressing this topic in the hospitals of Almadinah
Almunawwarah, Saudi Arabia. This study will add to the database of knowledge and attitudes on pain assessment among health care providers in Saudi Arabia. We aim to present data on the assessment of pain, knowledge of pain, and attitudes regarding pain among health care providers in Almadinah Almunawwarah, Saudi Arabia.

Methods. This exploratory cross-sectional study presented data on the knowledge of pain assessment and attitudes toward pain among health care providers in Almadinah Almunawwarah, Saudi Arabia. Knowledge of pain assessment was examined by administering the Knowledge and Attitudes Survey Regarding Pain (KASRP).

Sample and setting. A non-random sampling technique was used (convenience sample) of health care providers recruited from all the major hospitals in Almadinah Almunawwarah; King Fahad Hospital, Ohud Hospital, Alansar Hospital, Almeqat Hospital, and the Maternity Hospital. The data collection occurred between January and April 2014. The researchers went to all the departments in the aforementioned hospitals, and invited all the nurses and physicians who were present at that time to participate. Restricting the sampling to Almadinah Almunawwarah allowed researchers to have personal contact with hospital administrators in each of the targeted institutions, which was important to foster their cooperation. The researchers distributed and collected the surveys from participants. Sample size was determined by power analysis, using the following parameters: significance level of 0.05 , power of 0.95 , and 37 response variables. With 100 subjects, an effect size of 0.20 or larger could be attained. Therefore, we sought a sample size of at least 100 participants. The researchers contacted 300 nurses and physicians, only 114 questionnaires were returned. Nine questionnaires were rejected because they were incomplete. The participants filled out the questionnaires individually after they received written and verbal instructions.

Protection of human subjects. Research approval was obtained for the study from the Research Counsel of the College of Medicine, Taibah University, Almadinah Almunawwarah, Saudi Arabia. Participation was voluntary, and there was no penalty or loss of benefits for not participating in the study. Participants were assured of the confidentiality of their responses and their right not to participate or to withdraw from the study at any time. Data is stored in a secure location accessible only to the investigators and all data will be destroyed 3-5 years after dissemination of the findings.

Instrument. Ferrell and McCaffery ${ }^{10}$ developed the KASRP tool over several years to assess health care professionals' knowledge, and attitude related 
to pain. The KASRP was developed in 1987 and has been used frequently since then. ${ }^{10}$ The KASRP tool has been revised over the years to reflect changes in pain management practice. The KASRP is a 37 -item questionnaire, containing 21 true or false questions, and 16 multiple-choice questions. Its goal is to evaluate the attitudes and knowledge of caregivers toward pain. ${ }^{10}$ The KASRP asks questions on knowledge and attitude toward pain such as: basic pain physiology, pain assessment, pharmacology, non-pharmacologic interventions to relieve pain, and reliable indicators of the intensity of a patient's pain. Pain knowledge and attitude questions are closely related. The KASRP content was validated. Its content was established from current standards of major scientific authorities in the field of pain, such as the American Pain Society, the World Health Organization, and the National Comprehensive Cancer Network Pain Guidelines. The validity of KASRP was established by comparing results of different groups of caregivers including nurses at different levels and senior pain experts. The tool was recognized as discriminating between levels of expertise. Repeat testing in a continuing education class of staff nurses $(\mathrm{N}=60)$ showed test-retest reliability $(\mathrm{r}>0.80)$ and internal consistency reliability (alpha $r>0.70$ ) with KASRP's items. ${ }^{10}$

Data collection. Permission was obtained from the selected institutions and printed versions of KASRP were distributed to participants. A brief description of the study and an invitation to participate was provided verbally and in writing. After completing the paper and pencil survey, participants were asked to return the survey to the researcher. Participants were invited to convey problems with the procedure to the investigator so changes can be made in a timely manner to facilitate data collection.

Data analysis. Responses to the KASRP questionnaire items and the demographic questions were entered into IBM SPSS Statistics, version 20.0 (IBM Corp., Armonk, NY, USA) for analysis of descriptive statistics, and nonparametric tests. Mann-Whitney U and Kruskal-Wallis statistics were used to determine the relationship between demographic characteristics and participants' achievements on the KASRP.

Results. The aim of this study was to present data on knowledge and attitudes regarding pain assessment among health care providers in Almadinah Almunawwarah, Saudi Arabia. Using the KASRP, data was collected between January and April 2014 from 5 hospitals in Almadinah Almunawwarah. A total of 105 questionnaires were collected, the range of scores for the sample were from $18-94 \%$, mean 41.7 , and standard deviation 12.1. The study sample consisted of 75 females $(71.4 \%)$, and 30 males (28.6\%). Eighty-seven participants were nurses, and 18 were physicians. Thirty participants had $1-3$ years of experience $(28.6 \%) ; 23$ had 5-10 years of experience (21.9\%); 21 had 3-5 years' experience (20\%); 17 had less than one year experience (16.2\%); 13 participants had 10-20 years of experience (12.4\%), and one participant had more than 20 years of experience (1\%). According to the data reported: 59 participants had bachelor's degrees (56.1\%), 38 had associate or diploma degrees $(36.1 \%), 5$ hold master's degrees $(4.8 \%)$, and 3 have doctoral degrees $(2.9 \%)$. Respondents' ages were divided into 3 categories; the 26-40 year old age range accounted for the largest number of respondents with 60 or $57.1 \%$. The $20-25$ age range was next highest with $32(30.5 \%)$, followed by 41 and older with $13(12.4 \%)$. For the nurses group, 43 participants (49.4\%) were Saudi Arabians, and 44 participants $(50.6 \%)$ were non-Saudis. Sixty-eight participants (78.2\%) were female, and 19 participants $(21.8 \%)$ were male. Tables $1 \& 2$ show the educational level, and years of experience. For the physician's group, 6 participants (33.3\%) were Saudi Arabians, and 12 participants $(66.7 \%)$ were non-Saudis. Seven participants $(38.9 \%)$ were female, and 11 participants (61.1\%) were male. Tables $3 \& 4$ show the educational level, and years of experience. There were no significant differences in the passing scores across means of

Table 1 - Nurse participants' educational level among studied health care providers from Almadinah Almunawwarah, Saudi Arabia.

\begin{tabular}{lrr}
\hline Educational level & \multicolumn{1}{c}{$\mathbf{n}$} & \multicolumn{1}{c}{$(\%)$} \\
\hline Associate degree & 3 & $(3.4)$ \\
Diploma & 35 & $(40.2)$ \\
Bachelor & 48 & $(55.2)$ \\
Master & 1 & $(1.1)$ \\
Total & $\mathbf{8 7}$ & $(\mathbf{1 0 0 . 0 )}$ \\
\hline
\end{tabular}

Table 2 - Nurse participants' years of experience among studied health care providers from Almadinah Almunawwarah, Saudi Arabia.

\begin{tabular}{lcr}
\hline Years of experience & $\mathbf{n}$ & \multicolumn{1}{c}{$(\%)$} \\
\hline$<1$ year & 12 & $(13.8)$ \\
1-3 years & 28 & $(32.2)$ \\
3-5 years & 18 & $(20.7)$ \\
5-10 years & 21 & $(24.1)$ \\
$>10$ years & 8 & $(9.2)$ \\
Total & 87 & $(\mathbf{1 0 0 . 0})$ \\
\hline
\end{tabular}


Table 3 - Physician participants' educational level among studied health care providers from Almadinah Almunawwarah, Saudi Arabia.

\begin{tabular}{lrc}
\hline Educational level & $\mathbf{n}$ & $(\%)$ \\
\hline Bachelors degree & 11 & $(61.1)$ \\
Masters degree & 4 & $(22.2)$ \\
Doctorate degree & 3 & $(16.7)$ \\
Total & 18 & $(100.0)$ \\
\hline
\end{tabular}

Table 4 - Physician participants' years of experience among studied health care providers from Almadinah Almunawwarah, Saudi Arabia.

\begin{tabular}{lrr}
\hline Years of experience & $\mathbf{n}$ & $(\%)$ \\
\hline$<1$ year & 5 & $(27.8)$ \\
1-3 years & 3 & $(16.7)$ \\
3-5 years & 3 & $(16.7)$ \\
5-10 years & 2 & $(11.1)$ \\
$>10$ years & 5 & $(27.8)$ \\
Total & 18 & $(100.0)$ \\
\hline
\end{tabular}

Table 5 - Age group and assessment of pain passing rate among studied health care providers from Almadinah Almunawwarah, Saudi Arabia.

\begin{tabular}{lrr}
\hline Age groups & $\mathbf{n}$ & $(\%)$ \\
\hline $20-25$ & & \\
Fail & 27 & $(84.3)$ \\
Pass & 5 & $(15.7)$ \\
$26-41$ & & \\
Fail & 40 & $(65.5)$ \\
Pass & 21 & $(34.5)$ \\
$42-60$ & & \\
Fail & 7 & $(58.3)$ \\
Pass & 5 & $(41.7)$ \\
\hline
\end{tabular}

Table 6 - Years of experience and assessment of pain passing rate among studied health care providers from Almadinah Almunawwarah, Saudi Arabia.

\begin{tabular}{lrr}
\hline Years of experience & $\mathbf{n}$ & $(\%)$ \\
\hline$<1$ year & & \\
Fail & 13 & $(76.5)$ \\
Pass & 4 & $(23.5)$ \\
1-3 years & & \\
Fail & 26 & $(83.9)$ \\
Pass & 5 & $(16.1)$ \\
3-5 years & & \\
Fail & 12 & $(57.1)$ \\
Pass & 9 & $(42.9)$ \\
5-10 years & & \\
Fail & 16 & $(69.6)$ \\
Pass & 7 & $(30.4)$ \\
$>10$ years & & \\
Fail & 6 & $(50.0)$ \\
Pass & 6 & $(50.0)$ \\
\hline
\end{tabular}

nationality ( $p=0.179)$, departments where participants were working ( $p=0.286)$, years of experience $(p=0.150)$, and age of participants $(p=0.154)$. Tables $5 \& 6$ show the passing rate for both groups according to age group and years of experience.

Discussion. Assessing pain with a valid and reliable tool is essential and effective in pain management, because pain can affect the physical, emotional, and social function of the client. ${ }^{1}$ Effective pain management includes: interview, physical assessment, medication review, medical and surgical review, psychosocial review, and physical environment review. Through these processes, health care providers can determine the cause of pain and the effectiveness of treatment. ${ }^{5}$ Seventythree participants scored $44 \%$ or below on knowledge of pain $(69.5 \%)$, and 32 participants scored $45 \%$ and above $(30.5 \%)$. Findings suggest there is a significant knowledge deficit on pain and pain management. Moreover, only 6 participants (5.7\%) scored above $60 \%$, which is the accepted passing rate.

Study findings require immediate action from health care leaders to educate health care providers on pain and pain management, which is similar to Moceri and Drevdahl's ${ }^{11}$ findings. Additionally, respondents indicated that no protocols exist for pain assessment, and health care providers do not use a specific tool to assess pain. Overall, findings suggest that there are deficits in the knowledge of health care providers related to pain, and they hold inappropriate attitudes to caring for patients experiencing pain. Furthermore, several nurses' comments on the open-ended question indicated that pain assessment tools are inaccurate and not beneficial. Profoundly, the consequences of these deficits would lead to disregarding the patients' report of pain, which results in an impact on the effective treatment of patients' pain.

Findings indicated significant differences between male and female scores, male mean and median scores were 46.43, and 45.50; female mean and median scores were 39.81, and $38.0(p<0.05)$. Moreover, 14 males (46.7\%) scored 44\% and below, and 16 males (53.3\%) scored $45 \%$ and above. On the other hand, 59 females (78.7\%) scored $44 \%$ and below, and 16 females $(21.3 \%)$ scored $45 \%$ and above. Male health care providers were more knowledgeable than female health care providers regarding pain; further research should be conducted to investigate this matter.

There were also significant differences between physicians' and nurses' scores on pain knowledge: physicians' mean and median scores were 48 , and 46 ; nurses' mean and median scores were 40.31 , and 38 $(p<0.001)$. Six physicians $(31.6 \%)$ scored $44 \%$ and 
below, and 13 physicians (68.4\%) scored 45\% and above; 67 nurses $(77.9 \%)$ scored $44 \%$ and below, and 19 nurses $(22.1 \%)$ scored $45 \%$ and above.

Zanolin et $\mathrm{al}^{2}$ conducted a study to survey the knowledge and attitudes toward pain of Italian health care providers, and developed a valid instrument to assess nurses' and physicians' pain knowledge. ${ }^{2}$ Findings indicated a statistically significant difference $(p<0.001)$ in percentage between physicians $(56.5 \%)$ and nurses (51\%). Pain knowledge was best among physicians in anesthesiology, and emergency care, followed by doctors in general medicine and surgery.

Findings also suggest significant differences regarding pain treatment, and assessment based on the level of education $(p<0.009)$. Thirty-five participants with diploma and associate degrees in nursing (92.1\%) scored $44 \%$ and below, and 3 participants with diploma and associate degrees in nursing (7.9\%) scored 45\% and above; 33 participants with baccalaureate degrees in nursing (68.8\%) scored $44 \%$ and below, and 15 participants with baccalaureate degrees in nursing (31.2\%) scored $45 \%$ and above. One participant (from all disciplines) with a doctoral degree $(33.3 \%)$ scored $44 \%$ and below, and 2 participants with doctoral degrees $(66.6 \%)$ scored $45 \%$ and above. Thus, it is clear healthcare providers with higher education levels are more likely to have more knowledge on pain and more likely to apply practices toward treating pain

According to Wilson ${ }^{12}$ nurses have less knowledge of pain than physicians; however, nurse specialists have a more comprehensive knowledge base than the generalist nurses. Finally, a study by Hirsh et $\mathrm{al}^{13}{ }^{13}$ shed modest insight into health care professionals' decision-making processes, indicating biases may factor into decisions on pain recognition and treatment.

Duignan and Dunn ${ }^{14}$ categorized the barriers that prevent use of pain management in emergency departments to: healthcare system-related barriers, and health care provider-related barriers. Healthcare system-related barriers include lack of time, lack of nurses' knowledge, and regulatory issues. Health care provider-related barriers include staff attitudes, beliefs regarding analgesia, and under-assessment of pain.

Implications for practice. Courses on pain knowledge and practices should be offered immediately for health care providers in Almadinah Almunawwarah. Likewise, pain protocols should be implemented across healthcare organizations. The researchers suggest the verbal numeric pain scale should immediately be adopted for patients who are able to communicate verbally, as well as the nonverbal pain scale for patients who cannot communicate verbally. The verbal numeric pain scale can be printed on identification-card size paper and attached to all healthcare providers' identification cards.
Medical and nursing schools should revise and update curricula to ensure sufficient knowledge on pain is integrated in different student courses, and that students use evidence-based protocols to assess and manage pain.

Study limitations. This study provides a detailed account of knowledge, attitudes, and practices of health care providers in Almadinah Almunawwarah, on pain, but cannot be generalized to other regions of Saudi Arabia, or to other countries in the Middle East. This study included nurses and physicians only. We recommend this study be replicated in a larger sample of all health care providers across all healthcare sectors in Saudi Arabia.

In conclusion, this study identified important aspects regarding pain assessment knowledge, attitude, and practices of health care providers in Almadinah Almunawwarah, Saudi Arabia. This study demonstrates that there is a shortage in pain assessment knowledge and pain management in the study group. Most participants in this study require continuous education on pain assessment to increase their knowledge and enhance their practices regarding pain assessment and treatment. Furthermore, the findings of the study may add to the database of knowledge, attitude, and practice related to pain in Saudi Arabia. It is further hoped that these findings will be considered when developing specific educational programs for health care providers to better meet the needs of patients with pain.

\section{References}

1. Breivik H, Borchgrevink PC, Allen SM, Rosseland LA, Romundstad L, Hals EK, et al. Assessment of pain. Br J Anaesth 2008; 101: 17-24.

2. Zanolin ME, Visentin M, Trentin L, Saiani L, Brugnolli A, Grassi M. A questionnaire to evaluate the knowledge and attitudes of health care providers on pain. J Pain Symptom Manage 2007; 33: 727-736.

3. Institute of Medicine. Relieving pain in America: A blueprint for transforming prevention, care, education and research. [updated 2012 March 20; accessed 2014 January 1] Available from: http://www.iom.edu/Reports/2011/Relieving-Painin-America-A-Blueprint-for-transforming-Prevention-CareEducation-Research.aspx

4. International Association for the Study of Pain. IASP taxonomy. [updated 2012 May 22; accessed 2013 December 19]. Available from: http://www.iasp-pain.org/Taxonomy

5. Silverthorn D. Human physiology: An integrated approach. 7th ed. San Francisco (CA): Pearson/Benjamin Cummings; 2015.

6. Association of Critical-Care Nurses. Assessing pain in the critically ill adult. [updated 2013 May 1; accessed 2013 December 18]. Available from: http://www.aacn.org/wd/ practice/content/practicealerts/assessing-pain-critically-illadult.pcms?menu=practice

7. Pasero C, McCaffery M. Pain assessment and pharmacologic management. St Louis (MO): Mosby-Elsevier; 2010.

8. Topolovec-Vranic J, Canzian S, Innis J, Pollmann-Mudryj MA, McFarlan AW, Baker AJ. Patient satisfaction and documentation of pain assessments and management after implementing the adult nonverbal pain scale. Am J Crit Care 2010; 19: 345-354. 
9. Abdalrahim M. Postoperative pain assessment and management: the effects of an educational program on Jordanian nurses' practice, knowledge, and attitude [Doctoral Thesis]. Gothenburg (SE): University of Gothenburg; 2009. Available from: https://gupea.ub.gu.se/bitstream/2077/20316/4/ gupea_2077_20316_4.pdf

10. Ferrell B, McCaffery M. Knowledge and attitudes survey regarding pain. City of Hope Pain \& Palliative Care Resource Center. [updated 2014 July; accessed 2013 September 13]. Available from: http://prc.coh.org/Knowldege\%20\%20\&\%20 Attitude\%20Survey\%207-14.pdf
11. Moceri JT, Drevdahl DJ. Nurses' knowledge and attitudes toward pain in the emergency department. J Emerg Nurs 2014; 40: 6-12.

12. Wilson B. Nurses' knowledge of pain. J Clin Nurs 2007; 16: 1012-1020.

13. Hirsh AT, Jensen MP, Robinson ME. Evaluation of nurses' self-insight into their pain assessment and treatment decisions. J Pain 2010; 11: 454-461.

14. Duignan M, Dunn V. Barriers to pain management in emergency departments. Emerg Nurse 2008; 15: 30-34.

\section{Authorship entitlement}

Excerpts from the Uniform Requirements for Manuscripts Submitted to Biomedical Journals updated November 2003.

Available from www.icmje.org

The International Committee of Medical Journal Editors has recommended the following criteria for authorship; these criteria are still appropriate for those journals that distinguish authors from other contributors.

Authorship credit should be based on 1) substantial contributions to conception and design, or acquisition of data, or analysis and interpretation of data; 2) intellectual content; and 3) final approval of the version to be published. Authors should meet conditions 1, 2, and 3 .

Acquisition of funding, collection of data, or general supervision of the research group, alone, does not justify authorship.

An author should be prepared to explain the order in which authors are listed. 